\title{
Revisão da Maytenus ilicifolia Mart. ex Reissek, Celastraceae. Contribuição ao estudo das propriedades farmacológicas
}

\author{
Ralph Santos-Oliveira, ${ }^{*}, 1$ Simone Coulaud-Cunha, ${ }^{2}$ Waldeciro Colaço ${ }^{3}$ \\ ${ }^{1}$ Instituto de Engenharia Nuclear, Rua Hélio de Almeida 75, Cidade Universitária, Ilha do Fundão, \\ 21041-000 Rio de Janeiro-RJ, Brasil \\ ${ }^{2}$ Agência Nacional de Vigilância Sanitária, SIA, Trecho 5, Área Especial 57, 71205-050 \\ Brasília-DF, Brasil \\ ${ }^{3}$ Departamento de Energia Nuclear, Universidade Federal de Pernambuco, Av. Prof. Luiz Freire 1000, \\ Cidade Universitária, 50740-540 Recife-PE, Brasil
}

\begin{abstract}
RESUMO: A Maytenus ilicifolia Mart. ex Reissek, Celastraceae, vem sendo utilizada à muitos anos na terapêutica clínica, contudo suas propriedades farmacológicas continuam sob estudo. O objetivo desse trabalho é fornecer subsídios teóricos para o aprimoramento dos estudos, em especial os estudos clínicos, e assim estabelecer definitivamente as propriedades reais da Maytenus ilicifolia.

Unitermos: Maytenus ilicifolia, Celastraceae, estudos clínicos, propriedades farmacológicas.

ABSTRACT: : "Review of Maytenus ilicifolia Mart. ex Reissek, Celastraceae. Contribution to the studies of pharmacological properties". The Maytenus ilicifolia Mart. ex Reissek, Celastraceae has been used for many years as a medicine in the therapeutic in Brazil. However, the pharmacological action still under evaluation by many research centres. The aim of this study is stablish, based in clinical trials, the real properties of Maytenus ilicifolia.
\end{abstract}

Keywords: Maytenus ilicifolia, Celastraceae, clinical trials, pharmacological properties.

\section{INTRODUÇÃO}

A Maytenus ilicifolia Mart. ex Reisseké conhecida popularmente como "espinheira-santa", "cancerosa", "cancorosa-de-sete-espinhos" e "maiteno", dentre outros nomes (Lorenzi \& Matos, 2002; Brandão et al., 2006). Pertence à família Celastraceae possuindo 55 gêneros e 850 espécies espalhadas nas regiões trópicas e subtrópicas do mundo. No contexto brasileiro, onde seu crescimento é nativo a espécie $M$. ilicifolia é largamente utilizada na medicina popular (Mossi et al., 2004). O uso medicinal de M. ilicifolia é datado da década de 20 desde quando se tem algum registro escrito de sua utilização (Cunha et al., 2003).

Segundo o uso popular acredita-se que a $M$. ilicifolia possa combater várias doenças, dentre as quais podem-se destacar, gastrites e dispepsias. Possui ações tônicas, analgésicas, anti-sépticas, cicatrizantes, diuréticas e laxativas (Coimbra, 1958). Cipriani et al. (2004) corrobora tais afirmações, dizendo que é uma erva com ação contra úlcera e gastrite, por meio de seus estudos. Segundo Carlini \& Frochtengarten (1988), os primeiros estudos de eficácia terapêutica da $M$. ilicifolia foram realizados por Aluizio França, professor da
Faculdade de Medicina do Paraná, em 1922 que usou a M. ilicifolia em pacientes portadores de úlcera gástrica e relatou o sucesso do tratamento. Atualmente há estudos que afirmam que a $M$. ilicifolia apresenta, também, atividades antineoplásica e antimicrobiana (Ming et al., 1998; Pereira et al., 1992). Cipriani et al. (2004) relatam, ainda a descoberta e caracterização de arabinogalactanas, polímeros essenciais encontrados nas paredes celulares de plantas superiores, que possuem atividade imunológica. Esse fato pode ajudar a explicar a melhora dos quadros de câncer mediante sua aplicação, já que a maioria, senão a totalidade das drogas antineoplásicas, apresenta grande efeito imunossupressor, principalmente quando associadas a procedimentos cirúrgicos e antiinflamatórios esteroidais, como betametasona e dexametasona, prática comum nos protocolos antineoplásicos. No caso do tratamento de úlceras e gastrites, pode ser preparada tanto na forma de emplastros de suas folhas, decocto, por infusão, como na forma de chás e extratos (Lorenzi \& Matos, 2002).

\section{ECOLOGIA}

Segundo Magalhães (2004), a M. ilicifolia é encontrada, predominantemente na região Sul do Brasil, no 
interiordematas nativas e emmatas ciliares. Tempreferência por solos argilosos, porém bem drenados e com alto teor de matéria orgânica. Apresenta predileção por climas temperado e subtropical. Um fato importante que deve ser lembrado refere-se ao espaçamento de plantio, ou seja, à época de transplante da planta, para que esta permaneça com seus componentes em concentrações adequadas, sendo a melhor época para o transplante a primavera. Ele alerta ainda que a irrigação deve ser frequente, principalmente até os dois primeiros anos, devendo a planta ser cultivada preferencialmente à sombra. $\mathrm{O}$ não seguimento dessas práticas pode resultar em alterações fitoquímicas severas na planta, como as relatadas por Radomski \& Wisniewski (1998), que detectaram o aparecimento de grandes quantidades de taninos nas folhas da M. ilicifolia cultivada a pleno sol. Portanto, técnicas de plantio padronizadas e seguras devem ser preconizadas, principalmente quando se trata de espécies com uso terapêutico, já que alterações em seu ciclo de plantio podem resultar em alterações nas concentrações, ou até desaparecimento de constituintes, dentre eles os que possuem ação terapêutica, ou seja, o princípio ativo da planta, ou mesmo o aparecimento de substâncias indesejáveis.

\section{COMPOSIÇÃO QUÍMICA}

De acordo com Lima et al. (1969), os primeiros pesquisadores a se dedicarem aos estudos fitoquímicos da Maytenus spp., houve a demonstração da presença de vários grupos fitoquímicos, destacando-se os terpenóides, taninos, alcalóides, macrólideos e flavonóides, dentre outros (Coimbra \& Da Silva, 1958; Simões, 1986; Carlini \& Frochtengarten, 1988; Pereira et al., 1992; Alonso 1998; e Coulaud-Cunha et al., 2004; Estevam et al., 2009). A presença desses grupos foi posteriormente confirmada e seus potenciais terapêuticos são bastante conhecidos.

Dentre as substâncias estudadas e classificadas na Maytenus ilicifolia, pode-se citar vários grupos, dentre eles os terpenos (maitenina, tringenona, isotenginona II, congorosinas $\mathrm{A}$ e $\mathrm{B}$, ácido maitenóico), os triterpenos (friedelanol e friedelina), óleos essenciais (friedenelol), taninos, principalmente os gálicos (epicatequina, epigalocatequinaegalatodeepigalocatequina),glicolipídeos (monogalactosildiacilglicerol, digalactosildiacilglicerol, trigalactosildiacilglicerol, tetragalactosildiacilglicerol e sulfoquinovosildiacilglicerol) e, por último, os alcalóides (maiteina, maitanprina e maitensina) (Alonso, 1998; Carlini \& Frochtengarten, 1988; Mendes et al., 2006).

Ohsaki et al. (2004), em pesquisas mais recentes com M. ilicifolia, descreveram quatro novos triterpenóides, denominados por eles de maytefolinas A, B e C e uvaol-3cafeato.

\section{PROPRIEDADES}

Atividade gástrica
De acordo com alguns estudos (Carlini \& Frochtengerten, 1988, Ming et al., 1998; CoulaudCunha et al. 2004; Carvalho et al., 2008) a M. ilicifolia apresenta ação contra úlcera péptica e gastrite. CoulaudCunha et al. (2004) relatam que a ação da M. ilicifolia na úlcera péptica e gastrite envolve mais de um mecanismo de ação, ainda não conclusivamente elucidados, e não se deve somente a um princípio ativo específico, mas a diferentes fitocomplexos. Foi demonstrado por Pereira et al. (1993) e por Ming et al. (1998), que tanto os taninos, principalmente a epigalocatequina, quanto os óleos essenciais, em especial o fridenelol, são responsáveis por parte dos efeitos gastroprotetores. Carlini \& Frochtengarten (1988), por sua vez, em estudos realizados com o abafado da M. ilicifolia relatam que, quanto maior o tempo do tratamento, maior será a gastroproteção sem, entretanto, haver alterações no pH. Tal observação pode ser confirmada por Ferreira et al. (2004) que, em estudos com extrato aquoso liofilizado das folhas de M. ilicifolia em sapos, comprovou que esse possui efeito inibitório sobre os mediadores $\mathrm{H}_{2}$ da histamina nas células parietais; esses segundo Gilman (1989), quando estimulados, causam a ativação da adenililciclase, iniciando uma série de alterações morfológicas e bioquímicas complexas, que leva ao aumento da secreção gástrica, funcionando como um antagonista $\mathrm{H}_{2}$, além de inibir o efeito da gastrina. Foi demonstrado, ainda, que tanto a epigalocatequina (tanino) quanto o fridenelol (óleo essencial) são responsáveis por parte do efeito protetor da mucosa gástrica (Pereira et al., 1993; Ming et al., 1998).

A atividade antiulcerogênica foi inicialmente estudada em modelos experimentais, como o da aspirina, indometacina-padrão, reserpina e imobilização em baixas temperaturas. Carlini \& Frochtengarten (1988) demonstraram, pelo estudo desses modelos o abafado de planta e o extrato liofilizado apresentaram um marcante efeito gastroprotetor. Traçando-se um comparativo entre o abafado e o extrato liofilizado, esse último monstrouse bem mais potente que o primeiro, em relação ao efeito gastroprotetor, apresentando, em alguns casos, atividade maior que a cimetidina. Souza-Formigoni et al. (1991), em estudos realizados com a infusão de folhas, tanto por via oral como via intraperitoneal, utilizando como controles positivos a ranitidina e a cimetidina, demonstraram que a M. ilicifolia tem atividade comparável, superior em alguns casos, com as atividades dos medicamentos-controle. Mister se faz ressaltar que se observou nesse estudo um aumento no volume do suco gástrico e efeitos sobre o $\mathrm{pH}$ idênticos ao da cimetidina (Souza-Formigoni et al. 1991). Estudo realizado, utilizando a fração hexânica bruta da M. ilicfolia, ou seja, uma fração rica em hidrocarbonetos e triterpenos, apresentou resultados semelhantes aos obtidos em estudos anteriores, sendo a fração hexânica bruta ativa na concentração de $4 \mathrm{mg} / \mathrm{kg}$ de acordo com o modelo utilizado que, neste estudo, foi o da indução pela indometacina (Faleiros et al., 1992). Ming et al (1998) e 
Pereira et al., (1993) em estudos realizados, afirmam que a atividade antiulcerogênica deve-se, primordialmente, aos taninos e derivados da catequina. Estudos posteriores comprovaram que as ações antiulcerogênica do tanino e dos derivados da catequina são potencializadas pela presença dos componentes dos óleos essenciais, friedelina e fridelol, o que sugere que mais de um componente tem efeito gastroprotetor. A comprovação do efeito sinérgico existente entre os componentes da $M$. ilicifolia foi corroborada pelos estudos realizados por Queiroga (2000), que demonstraram que os taninos, quando utilizados separadamente nos modelos de úlcera induzida por indometacina, não apresentam atividade.

Carlini \& Frochtengarten (1998) relacionaram a ação da espinheira-santa com sua riqueza em taninos e a identificação da presença de epigalocatequina nos extratos utilizados. Demonstraram ainda, que vários taninos gálicos, dentre eles a epigalocatequina, inibem a ATPase de membrana potássio-dependente das células da mucosa gástrica responsáveis pela secreção de ácido clorídrico no estômago, esse mecanismo se processa por inibição competitiva. Estudos posteriores, como o de Murakami et al. (1992) e Annuk et al. (1999) comprovaram, que há uma inibição não competitiva, sugerindo dois locais de atuação distintos. Ainda de acordo com Murakami et al. (1992), o epigalocatequina-3-galato mostrou-se o composto mais ativo. Segundo Annuk et al. (1999) através de seus estudos, há um outro mecanismo de ação, relacionado contra a Helicobacter pylori, freqüentemente envolvida em quadros de inflamação e ulceração da mucosa gástrica. Esses estudos demonstraram que taninos gálicos de diferentes plantas medicinais possuíam ação bacteriostática contra a $H$. pylori in vitro. Tal ação devia-se principalmente à alteração na permeabilidade da membrana, levando a perdas de eletrólitos e água. Foi demonstrado, ainda, que alguns polifenóis relacionados aos taninos e flavonóides atuavam em relação à adesão da bactéria à mucosa gástrica, dificultando sua aderência e, portanto, sua fixação, desta forma impedindo sua ação patogênica.

Em outro estudo, dirigido por Mabe et al. (1999), diversos taninos purificados foram testados contra $H$. pilori. O mais ativo é o epigalocatequina-3-galato, cuja CIM (concentração inibitória mínima) foi de $8 \mu \mathrm{g} /$ ml. Nesse estudo foi também induzida uma infecção experimental por H. pylori em roedores Gerbillus. Tanto uma fração de taninos gálicos e catéquicos quanto a epigalocatequina-3-galato foram eficientes em erradicar a infecção, em todos os animais tratados, enquanto ela persistiu nos controles, sugerindo grande eficiência dos taninos in vivo. Houve igualmente uma redução significativa de achados inflamatórios, focos de hemorragia e ulceração, nos animais tratados. Estudos realizados por Ferreira et al. (1996) demonstraram que o extrato etanólico exibe atividade semelhante aos bloqueadores $\mathrm{H}_{2}$ como a cimetidina, inibindo o aumento da produção de $\mathrm{HCl}$ pelas células oxínticas do fundo gástrico, induzido pela histamina. Corroborando estudos anteriores, Iwa et al (2001), demonstraram que a epigalocatequina-3galato inibiu úlceras de estresse induzidas, em ratos, por imersão em água fria por tempo prolongado. Esse efeito foi bloqueado por adição de pró-oxidantes, sugerindo que a atividade antioxidante da epigalocatequina-3-galato participa de sua atividade antiulcerosa.

Recentemente, os efeitos antiulcerosos de $M$. ilicifolia foram reconfirmados por outros autores, inclusive na forma de extrato seco produzido por "spray-dryer" (Bersani-Amado et al. 2000; Gonzales et al., 2001).

\section{Atividade antineoplásica}

Em relaçãoàatividade antineoplásica, os primeiros estudos foram realizados no Instituto de Antibióticos da Universidade Federal de Pernambuco. Nesses estudos foram isolados compostos triterpenoídicos com atividade citotóxica, in vitro, contra células tumorias (Santana et al., 1971). Estudos posteriores identificaram que a $M$. ilicifolia possui, ainda, atividade antioxidante, com ação quelante para metais pesados, além de agir sobre diferentes radicais livres (Ho et al., 1992; Melo et al., 2001).

Em tumores experimentais, o extrato apresentou atividade inibitória sobre o sarcoma 180 , de $87,46 \%$ na dose de 2,2 mg/kg por via intra-peritonial, e a inibição de 58,76\% em sarcoma de Yoshida (Santana et al., 1971). Estudos utilizando maitensina, em testes in vitro, com diversas linhagens de células neoplásicas, demonstrou ação em células Leuk-P 138, CA 9KB e V79 (Kupchan, 1972; Kupchan \& Karim, 1976). O mecanismo de ação da maitensina ocorre pela inibição da polimerização da tubulina, proteína que forma os microtúbulos nas células. A inibição da formação de microtúbulos interfere na atividade dos centrômeros, organelas que formam o fuso acromático durante a divisão celular, impedindo assim a reprodução de células cancerosas (Alonso, 1998). De acordo com Zeng (1994), outros triperpenos, além da maintesina, também exibem atividade citotóxica sobre linhagens tumorais in vitro, como a friedelina e o friedelol. Os taninos epigalocatequina e epigalocatequina-3-galato inibem a incorporação de timidina marcada em células tumorais gástricas, o que indica uma interferência na sua capacidade de crescimento. Essas substâncias também inibem a liberação de TGF $\beta$ (transforming growth factor B), cuja atuação pode induzir o surgimento de células malignas, assim como inibir a transcrição de NF-Kappa-B, um gene cuja expressão se relaciona com a carcinogênese (Okabe et al., 1999). Em outro estudo, Yamane et al., (1995) testaram a epigalocatequina-3-galato e determinaram que essa inibia a indução de câncer gástrico pela $N$-metil- $N$ nitroguanida, em estômagos de ratos. Parte desse efeito foi atribuído à inibição da ornitina descarboxilase, enzima que forma radicais nitrila altamente genotóxicos e indutores de mutação. 


\section{Atividade antimicrobiana}

Estudos pioneiros como o de Lima et al. (1969) já demonstravam que a maitenina exibe forte atividade antimicrobiana contra várias bactérias Gram positivas. Tais efeitos foram corroborados com a demonstração que os extratos das folhas e raízes têm efeito antimicrobiano para vários patógenos, dentre eles Staphylococcus aureus e Streptococcus sp.

Estudos de Annuk et al. (1999) confirmaram que os taninos gálicos podem inibir o crescimento de bactérias pela modificação da permeabilidade da parede celular. Taninos derivados da catequina possuem atividade in vivo e in vitro contra H. pylori (Mabe et al., 1999). De acordo com Singh \& Dubey (2001), a friedelina e o friedelan3 - $\beta$-ol possuem atividade antimicrobiana in vitro sobre S. aureus, E. coli, e também contra o fungo Aspergillus niger.

\section{Atividade sobre o Sistema Nervoso Central}

Segundo Alonso (1998), o extrato de M. ilicifolia possui atividade sedativa, que potencializa (efeito sinérgico) o sono por barbitúricos, em camundongos.

\section{Atividade sobre a espermatogênese}

Montanari et al. (1998) desenvolveram estudos com extratos etanólicos de M. ilicifolia em ratos, para comprovar seu efeito sobre a espermatogênese. Esse tipo de estudo possui caráter primordial, uma vez que os maiores usuários dos produtos à base de M. ilicifolia como remédio para a gastrite e/ou úlcera, são homens em idade sexualmente ativa, que passam a desenvolver o quadro clínico devido ao stress diário. O teste consistiu em ministrar, intraperitonealmente, $200 \mathrm{mg} / \mathrm{kg} / \mathrm{dia}$ e $800 \mathrm{mg} /$ $\mathrm{kg} / \mathrm{dia}$ de extrato de M. ilicifolia liofilizado, em dois grupos, um com cinco e outro com seis ratos, respectivamente. Em seguida os testes de microscopia pertinentes aos procedimentos foram realizados. Os autores concluiram que não houve alteração na espermatogênese com o uso de M. ilicifolia.

\section{Atividade antioxidante}

Em estudos com M. ilicifolia, Mattei \& Carlini (1998) utilizaram extrato liofilizado, in vitro, para testar a sua capacidade de inibir o processo de lipoperoxidação. A atividade foi avaliada em homogenato de cérebros de ratos. $O Q_{1 / 2}$ (coeficiente de inibição referente à concentração necessária para diminuir em $50 \%$ a oxidação em presença do liofilizado) foi de $10,0 \mathrm{mg}$. O dado indicou que o extrato de M. ilicifolia exerce uma importante atividade antioxidante, especialmente inibindo a peroxidação lipídica. Em outro estudo, o extrato de M. ilicifolia exibiu maior poder antioxidante, contra sulfato de estanho, num modelo de mutação de Salmonella. Nesse experimento o extrato demonstrou capacidade quelante de metais pesados (Melo et al., 2001). A espinheira-santa possui cerca de 19\% de substâncias polifenólicas, sob forma de taninos não condensados derivados da catequina (Carlini \& Frochtengarten, 1988). Os derivados da catequina são potentes antioxidantes (mais potentes que a vitamina $\mathrm{C}$ ou E para inibir a oxidação in vitro), além de exibirem atividade sobre diferentes radicais livres, tais como o radical superóxido ou peróxido (Ho et al., 1992). O uso de derivados da catequina, obtidos em decocto por 30 dias, causa um aumento da atividade de enzimas antioxidantes endógenas como a SOD (superóxido dismutase), glutation peroxidase, glutation redutase e catalase em células de diversos órgãos (rins, pulmões e intestino) em humanos, sugerindo uma ação aos níveis celular e sistêmico (Khan et al., 1992). A atividade antioxidante dos derivados da catequina é maior sobre o tubo digestivo, inibindo a lesão de células da mucosa por radicais livres gerados no processo digestivo, evidenciada num modelo in vitro. Esse efeito relaciona-se também com uma ação antimutagênica, protegendo contra agentes genotóxicos como a MeIQX (3,8-Dimethyl-3H-imidazo[4,5-f]quinoxalin-2-amine) que pode induzir transformação maligna em células da mucosa intestinal. Foi evidenciada uma redução da mutagênese induzida pela epigalocatequina-3-galato no teste de AMES (Krul et al., 2001).

\section{Outros efeitos}

Os triterpenos, em especial a friedelina, possuem atividade antiinflamatória, reduzindo o edema induzido na pata de ratos pela carragenina (Shmizu \& Tomoo, 1994). Esses efeitos podem explicar, em parte, a ação analgésica encontrada por Gonzales et al. (2001) no teste de compressão da cauda de camundongos. Friedelana e outros triterpenóides do gênero Maytenus inibem a enzima aldose redutase; essa atividade é fraca nos compostos isolados, mas consistente na fração de triterpenos. Essa enzima é responsável pelo excesso de síntese de sorbitol em diabéticos, mecanismo implicado em complicações como a neuropatia periférica dessa doença (Chavez et al., 1998).

\section{Farmacocinética}

O modelo da farmacocinética da espinheira-santa é estudado por meio de seus taninos não condensados. Epigalocatequina marcada foi administrada por via oral, sendo os picos plasmáticos observados 60 min após a ingestão. Após uma dose de $500 \mathrm{mg} / \mathrm{kg}$ foram detectadas concentrações tissulares de $12,3 \mathrm{mmol}$ no plasma, $48,4 \mathrm{mmol}$ no fígado e $565 \mathrm{mmol}$ no intestino delgado (Nakagawa \& Miyazawa, 1997). Depois da administração oral de epigalocatequina-3-galato marcada com trítio, a ratos, cerca de $6 \%$ da dose é eliminada na urina e $24 \%$ 
nas fezes nas primeiras 24 horas. Uma segunda dose, dada duas horas após a primeira, eleva em cerca de 4 a 6 vezes a concentração no plasma e em alguns tecidos. Esses dados sugerem uma meia-vida longa e eliminação lenta da epigalocatequina-3-galato. No final das 24 horas, os animais foram sacrificados e essa substância pôde ser encontrada no rim, cérebro, fígado, pâncreas, intestino e ossos (Suganuma et al., 1998). Num outro estudo, foi avaliada a afinidade da epigalocatequina-3-galato às proteínas do soro. Após uma dose oral, a ratos, mais de $50 \%$ da epigalocatequina-3-galato, absorvida ligou-se à proteínas do plasma, especialmente a fibronectina, o fibrinogênio e a HRGP (Histidin Rich Glicoprotein), proteína plasmática com 74 kdaltons cuja função ainda é pouco conhecida (Sazuka et al., 1996).

\section{ESTUDOS CLÍNICOS}

A espinheira-santa foi submetida a diversas séries clínicas não controladas na primeira metade do século passado, conduzidas por vários autores. Se não possuem maior valor científico, essas séries servem para reforçar sua larga etnofarmacologia e construir a fama da planta como medicamento para úlceras e gastrites. Os estudos farmacológicos e clínicos, feitos a partir de 1988, possuem resultados concordantes com as experiências médicas e populares do passado, no tratamento de queixas dispépticas, e suportam sua eficácia e segurança terapêutica no tratamento de úlcera péptica e dispepsia (Costa et al., 1992).

\section{Úlcera péptica e gastrite}

Em 1988 foi realizado um estudo clínico no qual foi observado a eficácia da espinheira-santa no tratamento de dispepsia alta. Nesse, um estudo duplo cego, com 23 pacientes com diagnóstico de dispepsia alta não ulcerada, treze pacientes receberam, durante 28 dias, duas cápsulas contendo $200 \mathrm{mg}$ cada de liofilizado de abafado de M. ilicifolia e dez pacientes, receberam cápsulas com placebo (açúcar mascavo). Apenas um paciente do grupo que recebeu espinheira-santa não terminou o tratamento contra cinco pacientes do grupo placebo. O grupo que ingeriu o liofilizado de espinheira-santa apresentou melhora significativa, em relação ao grupo placebo, no que diz respeito à sintomatologia dispéptica global, e principalmente nos sintomas de azia e gastralgia. Não houve queixas de efeitos colaterais com o uso de, espinheira-santa (Carlini \& Frochtengarten, 1988; Geocze et al., 1988).

Num segundo estudo, considerando um grupo de vinte pacientes portadores de úlcera péptica, diagnosticados endoscopicamente, dez receberam duas cápsulas diárias com $200 \mathrm{mg}$ de liofilizado de espinheira-santa, e dez receberam placebo (açúcar mascavo). Devido ao número de desistências (cinco e quatro, respectivamente) e das cicatrizações de úlceras terem ocorrido também no grupo placebo, os resultados não se diferenciaram estatisticamente. Mesmo assim, os pacientes que usaram o extrato de espinheira-santa tiveram resultados clínicos e endoscópicos ligeiramente melhores que os que receberam placebos. Como é conhecida a dificuldade nos ensaios clínicos com agentes anti-úlcera, devido à grande reincidência de sintomas e à dificuldade de cicatrização, que ocorre em pacientes submetidos a placebo, é necessário pesquisar um maior número de pacientes que deverá ser observado por um período de tempo de 4 a 6 meses para que se possa ter uma resposta conclusiva sobre o efeito cicatrizante da espinheira-santa, já que os estudos de farmacologia pré-clínica são positivos (Carlini \& Frochtengarten, 1988; Geocze et al., 1988). Outra razão, que vem sendo apontada por alguns autores para o insucesso relativo desse estudo, é a dosagem $(7,5 \mathrm{mg} /$ $\mathrm{kg}$ ao dia de extrato seco) considerada baixa em relação aos resultados da farmacologia e à própria dosagem usada segundo a experiência clínica não controlada dos médicos do início do século, que indicavam de 25 a $50 \mathrm{mg} / \mathrm{kg}$ de extrato seco ao dia para úlcera péptica (Coimbra, 1994; Alonso, 1998).

\section{Câncer}

Em 1971 foi realizado um estudo clínico para avaliar as propriedades anticancerígenas do extrato de espinheira-santa, no Instituto de Antibióticos de Pernambuco. Nesse estudo, 25 pacientes portadores de neoplasias em estados avançados e resistentes a outros quimioterápicos foram tratados com $150 \mu \mathrm{g} / \mathrm{kg} / \mathrm{dia}$ e 450 $\mu \mathrm{g} / \mathrm{kg} / \mathrm{dia}$ de triterpenos extraídos de Maytenus sp. ricos em maiteina, por via endovenosa. $\mathrm{O}$ efeito terapêutico foi avaliado nos tumores de acordo com critérios propostos por Karnofsky: pressão sangüínea, pulso, respiração e condições gerais do paciente foram observadas durante e após a administração da droga; contagem de hemácias, hematócrito, concentração de hemoglobina, contagem global e diferencial dos leucócitos e contagem de plaquetas foram realizados semanalmente, bem como bilirrubina, dosagem de proteínas, transaminases, creatinina, glucose, uréia e fosfatase alcalina, e exame de urina. Foram realizados, quinzenalmente, exames radiográficos, além do exame clínico diário. Os mais objetivos resultados foram obtidos em quatro pacientes portadores de carcinoma epidermóide de pilares da amídala ou tonsila, bordo da base da língua e da laringe. Nesses pacientes houve uma redução das lesões de cerca de 40 a $60 \%$, por período de 15 a 25 dias, bem como o desaparecimento do sangramento. Quanto às respostas subjetivas, foi verificada melhora acentuada das dores, astenia e da anorexia. Para carcinomas de útero e de ovário, osteossarcoma e condrossarcom, a não foram verificados resultados satisfatórios. Dos sete casos de carcinoma epidermóide de útero, foram observados resultados subjetivos positivos, em dois casos. Quanto 
ao carcinoma de estômago, foi detectada uma melhora de $30 \%$ em um dos três casos tratados, com melhora do estado geral de dois pacientes. No linfoepitelioma com invasão para a órbita foi observada uma redução de 40\% (Santana et al., 1971).

Numa outra série clínica a maitenina extraída da Maytenus sp., agora purificada, foi usada em associação com outras substâncias anticancerígenas de origem natural, em onze pacientes com carcinoma baso-celular avançado. Todos os pacientes melhoraram clinicamente, apresentando reduções superiores a $50 \%$ do tamanho da lesão. A maitenina tem pouco potencial de irritação para a pele, mas sua ação foi considerada lenta. Um aspecto curioso relatado, pelos autores, é que as lesões continuaram a regredir por vários dias, mesmo após o uso tópico da suspensão da maitenina, sugerindo um prolongado efeito residual (Melo et al., 1974). Os triterpenos ficaram esquecidos por longos anos. Recentemente, contudo, surgiu um novo interesse no uso de triterpenóides quinometídicos, em especial da maitenina, como drogas para o tratamento do câncer, em função de sua baixa toxicidade e capacidade de potencializar outros quimioterápicos. Os estudos que estão sendo realizados, entretanto, não foram publicados porque visam o lançamento de novas drogas pela indústria farmacêutica (Corsino et al., 2000)

\section{Indicações clínicas}

Em consequência dos estudos clínicos realizados assim como dos aspectos de etnofarmacologia, foi concedido à "espinheira santa" diversas aplicações na clínica médica tradicional, particularmente, na dispepsia alta não ulcerosa, gastrite, ulcera péptica e na constipação intestinal. Em todas as indicações seu poder digestivo, cicatrizante, antiinflamatório e protetor da mucosa gástrica são os mais acentuados Costa et al., 1992; Carlini \& Frochtengarten, 1988; Coimbra \& Da Silva, 1958).

\section{Efeitos colaterais}

Não foram relatadas reações adversas sérias ou que coloquem em risco a saúde dos pacientes (Carlini \& Frochtengarten, 1988; Alonso, 1998). Um estudo de tolerância clínica foi feito com sete voluntários saudáveis que usaram o dobro da dosagem máxima relatada (200 $\mathrm{ml}$ a $10 \%$, ao dia) para o decocto de espinheira-santa, por 14 dias, sem o relato de qualquer efeito adverso ou sintoma subjetivo (Carlini \& Frochtengarten, 1988). Os estudos feitos com roedores sugerem que $M$. ilicifolia e M. aquifolium possuem excelente tolerância terapêutica e toxicidade muito baixa, afastando a possibilidade de efeitos tóxicos ou cumulativos, como conseqüência dos fármacos dessas espécies, mesmo os com atividade anticancerígena (Alonso, 1998).

\section{Reações adversas}

Nos 43 pacientes estudados foram descritas sensação de boca seca, náusea e gastralgia, em poucos voluntários, mas com melhora no decorrer do estudo. A incidência de efeitos adversos foi igual entre os grupos placebo e tratado, o que sugere que a incidência de efeitos adversos é muito baixa com a espinheira-santa (Carlini \& Frochtengarten, 1988).

\section{Contra-indicações}

\section{Gestação}

Em outras partes da América do Sul, a espinheira-santa é usada no controle de natalidade, como contraceptivo. De acordo com Montanari \& Bevilacqua (2002), administrando-se uma dose de $1000 \mathrm{mg} / \mathrm{kg} / \mathrm{dia}$ de extrato hidroalcoólico liofilizado, das folhas, via oral, a ratas entre o primeiro e o terceiro dias de gravidez (DOP - day of pregnancy), entre o quarto e o sexto DOP e entre o sétimo e o nono DOP, foi verificada uma diminuição da pré-implantação embrionária, mas não foram observados efeitos na implantação nem na organogênese, assim como efeitos teratogênicos. No tocante a atividade estrogênica do extrato, os resultados sugeriram uma interferência na parede uterina, dificultando a aderência do embrião. Sendo assim, a espinheira-santa teria uma atividade contraceptiva, e não teratogênica. Ressalta-se, entretanto, que a Resolução SES/RJ no. 1757 de 18 de fevereiro de 2002 contra-indica (no âmbito do Estado do Rio de Janeiro), em geral, durante o primeiro trimestre de gestação e lactação o uso interno de drogas vegetais medicinais, cujos estudos toxicológicos não estejam concluídos.

\section{Durante a amamentação}

Existem indícios que o uso de espinheira-santa cause redução do leite materno (Vieira \& Albuquerque, 1998). No anexo da Resolução SES/RJ no. 1757 de 18 de fevereiro de 2002 está contra-indicado o uso interno da espinheira-santa, durante a lactação, com base nos estudos de Brinker (1998) e Bringel et al. (1976), que demonstraram redução do leite materno.

\section{Toxicologia pré-clínica aguda}

O estudo de toxicologia aguda foi realizado em camundongose em ratos sob a forma de abafado e liofilizado. Quando administrados por via oral, agudamente, esses preparados não evidenciaram efeitos tóxicos, pois doses até 1600 vezes maiores que as utilizadas pelo homem não causaram mortes, não modificaram a atividade motora, não prejudicaram o desempenho no teste do rota-rod, não potencializaram o tempo de sono do pentabarbital além de revelarem não possuir efeitos analgésico, anticonvulsivante 
e neuroléptico. Tais resultados indicam que a espinheirasanta não possui efeito tóxico, quando administrada de forma aguda, e não possui efeitos depressores do SNC (Sistema Nervoso Central). A toxicidade dos abafados foi tal que não foi possível calcular-se a $\mathrm{DL}_{50}$; assim, doses extremamente elevadas de $\mathrm{C}_{800}-\mathrm{C}_{1800}(5,4 \mathrm{e} 10,8 \mathrm{~g} / \mathrm{kg})$ não revelaram efeito letal mesmo após sete dias de observação (Carlini \& Frochtengarten, 1988). Porém, em outro estudo realizado, camundongos que receberam a administração oral de $1360 \mathrm{mg} / \mathrm{kg}$ e $2750 \mathrm{mg} / \mathrm{kg}$ de Maytenus, doses bem maiores que as preconizadas clinicamente, apresentaram aumento da frequência urinária de defecação e piloereção, não apresentando diferença quando comparados com o grupo controle. Porém, com a administração intraperitoneal os resultados foram diferentes, pois se observou significativo efeito sedativo já nas doses de $680 \mathrm{mg} / \mathrm{kg}$ e potencialização do tempo de sono induzido por barbitúrico (Oliveira et al., 1991).

\section{Toxicologia pré-clínica crônica}

A toxicologia crônica foi testada em ratos e camundongos machos que receberam o abafado de $M$. ilicifolia por 2 a 3 meses, em doses 20 a 40 vezes maiores que a dose comumente usada por humanos, e 360 vezes maiores que a dose comumente administrada a ratos e camundongos. Os resultados mostraram que não houve alterações no peso, no comportamento, na temperatura e nos parâmetros bioquímicos séricos e hematológicos. Os ratos machos tratados durante dois meses não apresentaram redução da capacidade reprodutora, e os filhotes gerados desenvolveram-se normalmente. Nas fêmeas, o ciclo menstrual não foi alterado pela administração diária crônica, bem como a sua capacidade de reprodução. As proles, nascidas de fêmeas que receberam espinheira-santa durante toda a gravidez, tinham número e peso iguais aos das fêmeas-controle. Além disso, não se observou diferenças no desenvolvimento das proles e não foi observada a presença de lesões teratogênicas. Também, não foram detectadas alterações hematológicas nas séries branca, vermelha e nem no exame anatomo-patológico das vísceras desses animais (Carlini \& Frochtengarten, 1988).

\section{Toxicologia dos componentes}

Em 1971, outra pesquisa aponta a $\mathrm{DL}_{50}$ da maitenina como sendo $19,39 \mathrm{mg} / \mathrm{kg}$ por via intraperitoneal. Nesse mesmo estudo, a contagem de eritrócitos e leucócitos foi considerada normal. Os efeitos sobre o intestino isolado de cobaias e o coração de sapo in situ com $2 \mathrm{mg} / \mathrm{ml}$ não mostraram mudanças no seu estado fisiológico normal. A absorção e a excreção urinária não mostraram a presença do antibiótico (maitenina) até a $4^{\text {a. }}$ hora após a administração da droga.

Não houve alterações significativas na pressão arterial e na respiração. Foram evidenciados efeitos irritantes sobre a pele de ratos nas doses de 10 e de 12 $\mathrm{mg} / \mathrm{kg}$ quando essas foram injetados por via indradérmica (Santana et al., 1971).

\section{Tolerância clínica}

Foram realizados estudos farmacológicos e clínicos cujos resultados demonstram eficácia e segurança terapêutica da espinheira-santa nos casos de úlcera péptica e dispepsia e nas doses recomendadas (Costa et al, 1992). Em estudo clínico realizado em 1971, com o objetivo de investigar a atividade antineoplásica de Maytenus sp, 25 pacientes com neoplasias em estados avançados e resistentes a outros quimioterápicos, foram tratados com $150 \mu \mathrm{g} / \mathrm{kg} / \mathrm{dia}$ a $450 \mu \mathrm{g} / \mathrm{Kg} /$ dia de maiteina e.v. Entre os efeitos observados, o mais comum foi a irritação do endotélio vascular, determinando flebite no local da aplicação e produzindo também tromboflebite, impossibilitando, assim, o uso de doses mais fortes. Em 5\% dos casos observou-se albuminúria, porém sem sintomas dolorosos renais. No exame bioquímico do sangue não foi verificada nenhuma alteração. Não foram observadas, também, manifestações tóxicas, por parte do fígado, nem pelo sistema nervoso (Santana et al., 1971).

Posteriormente, em 1988, por meio de um projeto da Central de Medicamentos (CEME, 1998), a Escola Paulista de Medicina realizou um estudo de Toxicologia clínica (Fase I). Foi administrado a sete voluntários sadios, o abafado de M. ilicifolia, em dosagem correspondente ao dobro da utilizada comumente na etnofarmacologia, durante 14 dias. Os voluntários foram submetidos a exame físico, ECG (eletrocardiograma), dosagem bioquímica sérica, exame de urina, hematologia (séries branca e vermelha) e responderam a um questionário sobre efeitos colaterais no início do estudo, antes das ingestões diárias da planta e após o $1^{\circ}, 7^{\circ} \mathrm{e} 14^{\circ}$ dia do tratamento. Não foram observados resultados anormais que pudessem ser atribuídos ao uso da planta, apenas sintomas como boca seca, náusea, gastralgia em poucos voluntários, com melhora no decorrer do estudo. Além disso, os voluntários não tiveram dificuldade em ingerir o abafado e não relataram a ocorrência de outros efeitos colaterais. Os resultados mostraram que o abafado de espinheira-santa não é tóxico para o ser humano quando utilizado da forma que é utilizado na medicina popular (Carlini \& Frochtengarten, 1988). Não obstante, de acordo com os resultados dos estudos clínicos realizados com a espinheira-santa, em 43 pacientes, não foram observados efeitos indesejados durante o tratamento por 28 dias, com $400 \mathrm{mg}$ diários de liofilizado do abafado de espinheirasanta (Geocze et al., 1988).

\section{Superdosagem}

De acordo com Carlini \& Frochtengarten (1988) posteriormente confirmado por Alonso (1999), não há 
relatos de superdosagem da espinheira-santa. Schvartsman (1992) entretanto, contrapõe que com doses excessivas, as plantas ricas em taninos podem causar irritação da mucosa gástrica e intestinal, gerando cólicas intestinais e diarréia.

\section{Adulterações}

No mercado informal é fácil encontrar espinheirasanta à venda. No entanto, pode-se observar, principalmente nas feiras livres, que a espécie oferecida não é M. ilicifolia e sim Sorocea bomplandi Bailon (Moraceae), uma das espécies mais utilizadas na adulteração da espinheirasanta. Essa freqüência fez com que diversos pesquisadores estudassem a espécie $S$. bomplandi e, por meio de pesquisas fitoquímicas e farmacológicas, verificaram a presença de alguns flavonóides e ações analgésica e anti-ulcerogênica semelhantes à da M. ilicifolia (Calixto et al., 1993; Gonzales et al., 2001). No entanto, não existem estudos comparativos sobre a eficácia das duas espécies, nem estudos que assegurem a falta de toxicidade crônica de $S$. bomplandii, o que pode se tornar um risco para as pessoas que inadvertidamente consomem essa espécie, pensando se tratar de espinheira-santa. Estudos recentes, já tornam possível a diferenciação macro e microscópica entre essas espécies, além da caracterização por marcadores químicos, o que se torna um potencial aliado para o controle de qualidade de produtos fitoterápicos (Jacomassi, 2000).

\section{REFERÊNCIAS}

Alonso JR. 1998. Tratado de fitomedicina bases clinicas y farmacológicas. Buenos Aires, Isis Ediciones SRL, p. 828-834.

Annuk H, Hirmo S, Türi E, Mikelsaar M, Arak E, Wadstrom $\mathrm{T}$ 1999. Effect on cell surface hydrophobicity and susceptibility of Helicobacter pylori to medicinal plant extracts. FEMS Microbiol Lett 172: 41-45.

Bersani-Amado CA, Massao LB, Bacio SR 2000 Anti-ulcer effectiveness of Maytenus aquifolium spray dried extract. Phytother Res 14: 543-545.

Brandão MGL, Cosenza GP, Moreira RA, Monte-Mor RLM 2006. Medicinal plants and other botanical products from the Brazilian Official Pharmacopoeia. Rev Bras Farmacogn 16: 408-420.

Bringel AS, Fong H, Farnworth N 1976. Antifertility screening of selected plants in female rats. L Loydia 39: 475.

Brinker F 1998. Herb contraindications and drug interactions. Sandy, Oregon. Ecletic Medical Publications, p. 91-92, 174-183.

Calixto JB, Messana I, Della Monache F, Ferrari F, Bisognin T, Yunes RA 1993. Pharmacological analysis of the methanolic extract and sorocein A, a new Diels-Alder compound isolated from the roots of Sorocea bonplandii Bailon in the isolated rat uterus and guinea pig ileum. Gen Pharmacol 24: 983-989.

Carlini EA, Frochtengarten ML 1988. Toxicologia clínica (Fase
I) da espinheira-santa (Maytenus ilicifolia).BrasíliaDistrito Federal, p. 67-73.

Carvalho ACB, Balbino EE, Maciel A, Perfeito JPS 2008. Situação do registro de medicamentos fitoterápicos no Brasil. Rev Bras Farmacogn 18: 314-319.

Chavez H, Estevez-Braun A, Ravelo AG, Gonzalez AG 1998. Friedelane triterpenoids from Maytenus macrocarpa. $J$ Nat Prod 61: 82-85.

Cipriani TR, Mellinger CG, Gorin PAJ, Iacomini M 2004. An arabinogalactan isolated from the medicinal plant Maytenus ilicifolia. J Nat Prod 67: 703-706.

Coimbra R 1994. Manual de fitoterapia. Belém: Ed. CEJUP, p. 130.

Coimbra R, Da Silva ED 1958. Notas de fitoterapia. Rio de Janeiro: Laboratório Clínico Silva Araújo, p. 32-33.

Corsino J, De Carvalho PR, Kato MJ, Latorre LR, Oliveira OMMF, Araujo AR, Bolzani VS, França SC, Pereira MAS, Furlan M 2000. Biosynthesis of friedelane and quinonemethide triterpenoids is compartmentalized in Maytenus aquifolium and Salacia campestris. Phytochemistry 55: 741-748.

Costa MA, Andrade CLZ, Vieira RF, Sampaio FC 1992. Plantas e saúde - guia introdutório a fitoterapia. Brasília: Secretaria de Saúde do Distrito Federal, p. 63-5.

Coulad-Cunha S, Oliveira RS, Waissmann W 2004. Venda Livre de Sorocea Bompladii Bailon como Espinheira Santa no Município do Rio de Janeiro. Congresso Ibero-Americano de Plantas Medicinais, Angra dos Reis.

Cunha SC 2003. Ação farmacológica da espinheira-santa: usos e precauções. Lavras, 100 p. Monografia de Especialização, Universidade Federal de Lavras..

CEME 1988. Ministério da Saúde. Estudo de ação antiúlcera gástrica de plantas brasileiras (maytenus ilicifolia "espinheira santa" e outras).

Estevam CS, Cavalcanti AM, Cambui EVF, Araújo Neto V, Leopoldo PTG, Fernandes RPM, Araujo BS, Porfírio Z, Sant'Ana AEG 2009. Perfil fitoquímico e ensaio microbiológico dos extratos da entrecasca de Maytenus rigida Mart. (Celastraceae). Rev Bras Farmacogn 19: 299-303.

Faleiros ICF 1992. Efeito antiulcerogênico de frações hexânicas das folhas de Maytenus ilicifolia (espinheira santa). $22^{\circ}$ Simpósio de Plantas Medicinais do Brasil. Curitiba.

Ferreira PM, Oliveira CN, Zhao-Hua W, Oliveira AB, Vieira MAR 1996. Envolvimento da histamina no mecanismo de ação do extrato bruto das folhas de Maytenus ilicifolia (espinheira santa) sobre a secreção gástrica de ácido. $14^{\circ}$ Simpósio de Plantas Medicinais do Brasil. Florianópolis.

Ferreira PM, Oliveira CN, Oliveira AB, Lopes MJ, Alzamora F, Vieira MAR 2004. A lyophilized aqueous extract of Maytenus ilicifolia leaves inhibits histamine-mediated acid secretion in isolated frog gastric mucosa. Planta 219: 319-324.

Geocze S, Vilela MP, Chaves BDR, Ferrari AP 1988. Tratamento de pacientes portadores com dispepsia alta ou úlcera 
péptica com preparações de espinheira-santa (Maytenus ilicifolia). Publicação CEME, PPPM no. 2, p.75-87.

Gilman AG 1989.Transmembrane signalling, Gproteins, and adenylyl cyclase. Harvey Lect 90: 153-172.

Gonzalez FG, Portela TY, Stipp EJ, Di Stasi, LC 2001. Antiulcerogenic and analgesic effects of Maytenus aquifoliuim, Sorocea bomplandii and Zolernia ilicifolia. J Ethnopharmacol 77: 41-47.

Ho CT, Chen Q, Shi H 1992. Antioxidative effects of polyphenol extract prepared from various chinese herbs. Prev Med 21: 520-525.

Iwa K, Onodena A, Matsue H 2001. Antioxidant activity and inhibitory effect of gamazumi (Viiburnum dilatatum Thumb.) on oxidative damage induced by water imersion restraint stress on rats. Int J Food Sci Nutr 52: 443-451.

Jacomassi E 2000. Morfo-anatomia comparativa, caule e folha, de Maytenus ilicifolia, Maytenus aquifolia (Celastraceae) e Sorocea bonplandii (Moraceae). São Paulo, 89 p. Dissertação de Mestrado, Universidade Estadual Paulista Júlio de Mesquita Filho.

Khan SG, Katiyar SK, Agarwal R, Mukhtar H 1992. Enhancement of antioxidant and fase II enzymes by oral feeding of green tea polyphenols in drinking water to SKH-1 hairless mice: Possible role in cancer chemoprevention. Cancer Res 52: 4050-4052.

Krul C, Luiten-Schuite A, Tenfelde A Ommem B, Verhagen H, Havenaar R 2001. Antimutagenic activity of green tea and black tea extracts studied in a dynamic in vitro gastrointestinal model. Mutat Res 474: 71-85.

Kupchan SM 1972. Recent advances in the chemistry of tumor inhibitors of plant origin, swain, tony. Plants in the Development of Modern Medicine Harvard, Harvard University Press, p. 261-278.

Kupchan SM, Karim A 1976. Tumor inhibitors. 114. aloe emodin: antilukemic principle isolated from Rhamnus frangula $\mathrm{L}$. Lloydia 39: 223-224.

Lima OG, Coelho JSB, Weigert E, D’Albuquerque IL, Souza MAM. 1969. Substâncias antimicrobianas de plantas superiores. Rev Inst Antibioticos 9: 17-25.

Lorenzi H, Matos FJA 2002. Plantas medicinais no brasil nativas e exóticas. São Paulo: Instituto Plantarum, p. 120-122.

Mabe K, Yamada M, Oguni I 1999. In vitro and in vivo activities of tea catechins aigainist Helicobacter pylori. Antimicrob Agents Chemother 43: 1788-1791.

Magalhães PM 2004. Agrotecnologia para o cultivo da espinheira santa. artigo completo. CPQBA-UNICAMP, [on line]. Disponível na Internet via URL: <http://www.cpqba. unicamp.br/plmed/artigos/agroespsant.htm $>$.

Mattei R, Carlini ELA 1998. Propriedades anti-oxidantes (lipoperoxidação) da Pfaffia glomerata, Heteropteris aphrodisiaca, Maytenus ilicifolia: estudo in vitro. $15^{\circ}$ XV Simpósio de Plantas Medicinais do Brasil, Águas de Lindoia.

Melo AM, D'Albuquerque IL, Lacet Y 1974. Primeiras observações do uso tópico de primina, plumbagina e maitenina em pacientes com câncer de pele. Rev Inst Antibioticos 14: 9-16.

Melo SF, Soares SF, Costa RF, Da Silva CR, Oliveira MBN, Bezerra RJAC, Caldeira-de-Araujo A, Bernardo-Filho M 2001. Effect of the Cymbopogon citratus, Maytenus ilicifolia and Baccharis genistelloides extracts against the stannous chloride oxidative damage in Escherichia coli. Mutat Res 496: 33-38.

Mendes BG, Machado MJ, Falkenberg M 2006. Triagem de glicolipídios em plantas medicinais. Rev Bras Farmacogn 16: $568-575$.

Ming LC, Castro DM, Delachiave ME 1998. Plantas medicinais aromáticas e condimentares. Botucatu: Universidade Estadual Paulista.

Montanari T, De Carvalho JE, Dolder H 1998. Effect of Maytenus ilicifolia Mart. Ex Reiss on epermatogenesis. Contraception 57: 335-339.

Montanari T, Bevilacqua E 2002. Effect of Maytenus ilicifolia Mart. on pregnant mice. Contraception 65: 171-175.

Mossi AJ, Cansian RL, Carvalho AZ, Dariva C, Oliveira JV, Mazutti M, Filho IN, Echeverrigaray S 2004. Extraction and characterization of volatile compounds in maytenus ilicifolia, using high-pressure $\mathrm{CO}_{2}$. Fitoterapia 75: 168178.

Murakami S, Muramatsu M, Otomo S 1992. Gastric $\mathrm{H}^{+} \mathrm{K}\left({ }^{+}\right)$ ATPase inhibition by catechins. J Pharm Pharmacol 44: 926-928.

Nakagawa K, Miyazawa T 1997. Absortion and distribution of tea catechin (-)-epigallocatechin-3-gallate in the rat. $J$ Nutr Sci Vitaminol 43: 679-684.

Ohsaki A, Imai Y, Naruse M, Ayabe SI, Komiyama K, Takashima J 2004. Four new triterpenoids from Maytenus ilicifolia. J Nat Prod 6: 469-471.

Okabe S, Ochiai Y, Aida M, Park K, Kim SJ, Nomura T, Suganuma M, Fujiki H 1999. Mechanistic aspects of green tea as a cancer preventive: effect components on human stomach cell lines. Jpn J Cancer Res 90: 733-739.

Oliveira MG, Monteiro MG, Macaubas C, Barbosa VP, Carlini EA 1991. Pharmacologic and Toxicologic effects of two Maytenus species in laboratory animals. $J$ Ethnopharmacol 34: 29-41.

Pereira RR, Pizzollati MG, De Lima TCM 1992. Ação do extrato de Velozia stipitata sobre úlceras gástricas experimentais em ratos. $12^{\circ}$. Simpósio de Plantas Medicinais do Brasil, Curitiba.

Pereira AMS, Rodrigues DC, Cerdeira RM, França SC 1993 Isolamento de metabólitos de maytenus associadas à ação anti-úlcera gástrica. $12^{\circ}$. Simpósio de Plantas Medicinais do Brasil, Curitiba.

Queiroga CL, Silva GF, Dias PC, Possenti A 2000. Evaluation of the anti-ulcerogenic activity of friedelan-3-beta$\mathrm{ol}$ and friedelin isolated from Maytenus ilicifolia M. (Celastraceae). J Ethnopharmacol 72: 465-468.

Radomski MI, Wisniewski C 1998. Teores de elementos químicos hidrossolúveis em folhas e ramos de Maytenus ilicifolia Mart. $15^{\circ}$ Simpósio de Plantas Medicinais do Brasil, São 
Paulo.

Santana CF 1971. Estudos farmacodinâmicos e histopatológicos da Maitenina. Rev Inst Antibioticos 11: 61-67.

Santana CF, Asfora JJ, Cotias CT 1971. Primeiras observações sobre o emprego da maitenina em pacientes cancerosos. Rev Inst Antibioticos 11: 37-49.

Sazuka M, ItoiT, Suzuki Y, Odanis S, Koide T, Isemura M 1996. Evidence for the interaction between (-)-epigallocatechin gallate and human plasma proteins fibronectin, fibrinogen and histidin rich glycoprotein. Biosc Biotechnol Biochem 60: 1317-1319.

Schvartsman S 1992. Plantas venenosas e animais peçonhentos. São Paulo, Savier, 288 p.

Shimizu M, Tomoo T 1994. Anti-inflamatory constituents of topicaly applied drugs $\mathrm{V}$ : constituents with antiinflamatory from Aoki (Aukuba japonica Thumb.). Biol Pharm Bull 17: 665-667.

Simões CMO, Mentz LA, Schenkel EP, Irgang BE, Stehmann JR 1986. Plantas da medicina popular no Rio Grande do Sul. Porto alegre: Editora Universidade Federal do Rio Grande do Sul.

Singh B, Dubey MM 2001. Estimation of triterpenoids from Heliotropium maifolium Kohen ex Retz in vivo and in vitro: antimicrobial screening. Phytother Res 15: 231234.

Souza-Formigoni ML, Oliveira MG, Monteiro MG, SilveiraFilho NG, Braz S, Carlini EA 1991. Antiulcerogenics effects of two Maytenus species in laboratory animals. $J$ Ethnopharmacol 34: 21-27.

Suganuma M, Okabe S, Oniyama M, Tada Y, Ito H, Fujiki H 1998. Wide distribution of $[3 \mathrm{H}]$ (-)-epigallocatechin gallate a cancer preventive polyphenol in mouse tissue. Carcinogenesis 19: 1771-1776.

Vieira LS, Albuquerque JM 1998. Fitoterapia tropical - manual de plantas medicinais. Pará, p. 113-114.

Yamane T, Takahashi T, Kuwata K, Oya K, Inagake M, Kitao Y, Suganuma M, Fujiki H 1995. Inhibition of $N$-methyl$N$ '-nitro- $N$-nitrosoguanidine-induced carcinogenesis by (-)-epigallocatechin gallate in the rat glandular stomach. Cancer Res 55: 2081-2084.

Zeng CQ 1994. Cytotoxic triterpenoids and flavonoids from Artemisia annua. Planta Med 60: 54-57. 